\title{
The Perceptual Basis of Vast Space
}

\author{
Roberta L. Klatzky ${ }^{1}$ - William B. Thompson ${ }^{2} \cdot$ Jeanine K. Stefanucci $^{2}$ • \\ Devin Gill ${ }^{2}$ D. Kevin $\mathrm{McGee}^{2}$
}

Published online: 20 March 2017

(C) Psychonomic Society, Inc. 2017

\begin{abstract}
Vast" is a word often applied to environmental terrain that is perceived to have large spatial extent. This judgment is made even at viewing distances where traditional metric depth cues are not useful. This paper explores the perceptual basis of vast experience, including reliability and visual precursors. Experiment 1 demonstrated strong agreement in ratings of the spatial extent of two-dimensional (2D) scene images by participants in two countries under very different viewing conditions. Image categories labeled "vast" often exemplified scene attributes of ruggedness and openness (Oliva \& Torralba, 2001). Experiment 2 quantitatively assessed whether these properties predict vastness. High vastness ratings were associated with highly open, or moderately open but rugged, scenes. Experiment 3 provided evidence, consistent with theory, that metric distance perception does not directly mediate the observed vastness ratings. The question remains as to how people perceive vast space when information about environmental scale is unavailable from metric depth cues or associated scene properties. We consider possible answers, including contribution from strong cues to relative depth.
\end{abstract}

Keywords Perception $\cdot$ Scene $\cdot$ Depth $\cdot$ Space $\cdot$ Vast $\cdot$ Awe

Roberta L. Klatzky

klatzky@cmu.edu

1 Department of Psychology, Carnegie Mellon University, Pittsburgh, PA 15213, USA

2 University of Utah, Salt Lake City, UT, USA

\section{Introduction}

Try to recall when you stood in a "vast" space. You might imagine yourself at the Grand Canyon, or staring up at the stars, or perched at the ocean's edge. Although an object like the Colossus of Rhodes or an edifice like the Empire State building may be very large, when "vast" comes to mind it often is in relation to scenes: arrangements of objects and surfaces external to the viewer, generally in natural environments. What vast scenes share is that they extend to very far distances, seemingly without limit. Beyond what appears to be a common phenomenological experience, we know little of vastness as a psychological construct; for example, what cues induce it, how reliably they operate, and how it is related to other perceptual variables.

One possibility is that vastness corresponds to an extreme level of perceived depth. If so, the underlying percept cannot specify metric distance, as the traditional cues used to understand metrically scaled depth are uninformative for the very conditions in which spaces are called vast. The effectiveness of most visual depth cues drops off rapidly with distance from the observer, such that beyond 100 meters or so, metric depth estimates are not well supported, if supported at all (Cutting \& Vishton, 1995; Loomis \& Knapp, 2003). For example, the depth cue of binocular convergence relies on differences in the angles formed by the intersecting lines of sight to a target. Because the magnitude of the intersection angle varies inversely with target distance, angles become more similar as distance increases; as a result, the effective range of the convergence cue is only about 2 meters. Most of the pictorial cues to depth, such as height in the plane or familiar size, also fail to convey vast distances in metric terms. Linear perspective cues do not specify environmental scale unless the viewpoint is known. The cue of occlusion is present whenever objects overlap in the field of view, but offers only a 
binary cue to closer/farther. Aerial perspective arises at long distances, but varies unpredictably with moisture and atmospheric contaminants.

Given this limited range of cue utility, estimates of depth in metric terms seem unlikely to account for the sense of vastness that arises from the perception of extensive spaces. An alternative hypothesis is that the sense that a space is vast is a byproduct of mechanisms specialized for scene interpretation. Scene-specific features, such as natural and large, are extracted rapidly (Greene \& Oliva, 2009; Joubert et al., 2007), presumably by mechanisms operating at a more abstract level than those giving rise to metric depth (Henderson \& Hollingworth, 1999).

The percept of vastness may be related, in particular, to two variables based on the view-specific boundaries that structure the shape around a scene. As initially proposed by Oliva and Torralba (2001), openness is inversely related to the number of boundary elements, and ruggedness is inversely related to the extent to which contours arise obliquely from the ground plane to obscure the horizon. These variables are not independent, in that extremely open scenes are intrinsically non-rugged, but there are ranges where co-variation is not so constrained. Quantitative estimates of openness and ruggedness were derived for a set of scene images by first subjectively scaling the images according to category (e.g., designating openness as decreasing from coastline scenes to forests; ruggedness decreasing from mountains to fields). Notably, this analysis suggests a relation between these properties and vastness, as mountains and oceans are seen across the large distances associated with vast space. The researchers further showed that openness and ruggedness instantiate specific patterns of spatial frequency, by using a machine algorithm to classify the scaled values from spectral parameters and extracting a best-fit regression with spectral templates as predictors.

The present research constitutes an initial attempt to further our understanding of the perception of vast space and the relation of vastness to known scene properties. Experiment 1 - conducted in two countries using different displays - tested whether people could reliably assign subjective magnitudes representing the vastness of 2D photographic images. Variations across scene categories in the magnitude judgments, along with participants' recollection of experiences in vast spaces, suggest that vastness follows categorical distinctions like those used to scale the properties of openness and ruggedness (Oliva \& Torralba, 2001). Experiment 2 directly investigated how these variables are related by using separately obtained ratings of openness and ruggedness as predictors of the vastness magnitudes for the same photographs. Experiment 3 assessed the extent to which judgments of metric distance both predict vastness magnitudes and are themselves predicted by openness and ruggedness.

\section{Experiment 1: Subjective ratings of vastness}

\section{Method}

\section{Participants}

Thirty-one (16 females, 15 males) University of Utah college students (mean age $=23$ years) participated for course credit in the United States and 20 (10 females, 10 males) Tübingenarea residents (mean age $=28$ years) participated for 5 Euros compensation in Germany. All participants gave written consent. The sample sizes were based on conventions for standard psychophysical experiments. We targeted 20 participants, but all who were recruited were included in the data, and no analysis preceded the cessation of data collection.

\section{Materials and apparatus}

The stimuli were 134 full-color images of natural outdoor environments, ranging from close views of vegetation to mountains and beaches, which were gathered from pixabay.com and deviantart.com. Examples are shown in Fig. 1.

In Utah, seated participants viewed the images on an Asus PA246Q monitor, where they subtended a visual angle of approximately $15^{\circ}$ wide $\times 10^{\circ}$ high. In Germany, participants viewed the images on a large screen, wide field-of-view projection system from a specified standing point, where they subtended a visual angle of approximately $60^{\circ}$ wide $\times 42^{\circ}$ high .

Separately, the pictures were assigned by ten, University of Utah students and faculty to one of eight categories of scene

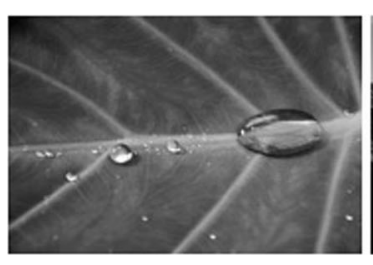

Fig. 1 Examples of stimulus photographs (originally in color). The leaf and shore images were retrieved from Pixabay. The mountain image was retrieved from DeviantArt, Siegle, Livesey, \& Browne (2005),
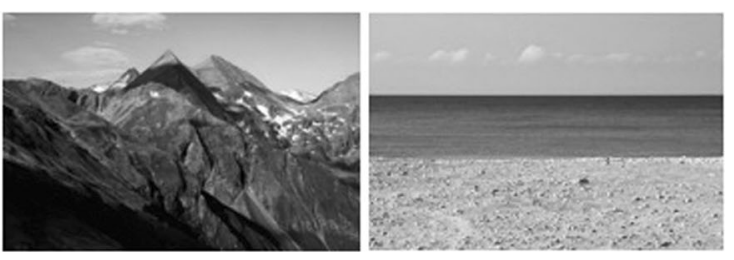

http://creativecommons.org/licenses/by/3.0/, http://austriaangloalliance. deviantart.com/art/Austrian-Peaks-328637716 
content. A reliability analysis found a Finn coefficient (used for categorical ratings; Finn, 1970) of 0.76, $F($ inf, 1179) $=$ $4.17, p<0.001$. In further analyses, each image was categorized by its consensus assignment, except for eight pictures where no consensus emerged.

\section{Procedure}

Participants were told that the experiment investigated cues in a visual scene that might produce the feeling that the depicted space was large. To orient them to the subsequent judgments, they were initially asked to give a description of a time when they felt that they had been in a vast space, including their vantage point. They were told (English version) "a space is vast if it seems to extend without limits away from you, making you feel like a small element within the space." They then transitioned to a rating task, where they were shown a series of images under the instructions, "Using a scale of 0-100, please judge the image in terms of how strongly you feel that the space depicted in the scene feels large." (Large was used in preference to vast, because it is unambiguous in English and has a direct counterpart in German.) Instructions were onscreen in the United States and oral in Germany. Three images were shown as practice. Participants were told to try to use the full rating scale, while being consistent in how they used it across comparable images. There was no time limit.

\section{Results}

Figure 2 shows average ratings for each of the 131 nonpractice images for the Germany and U.S. participants.

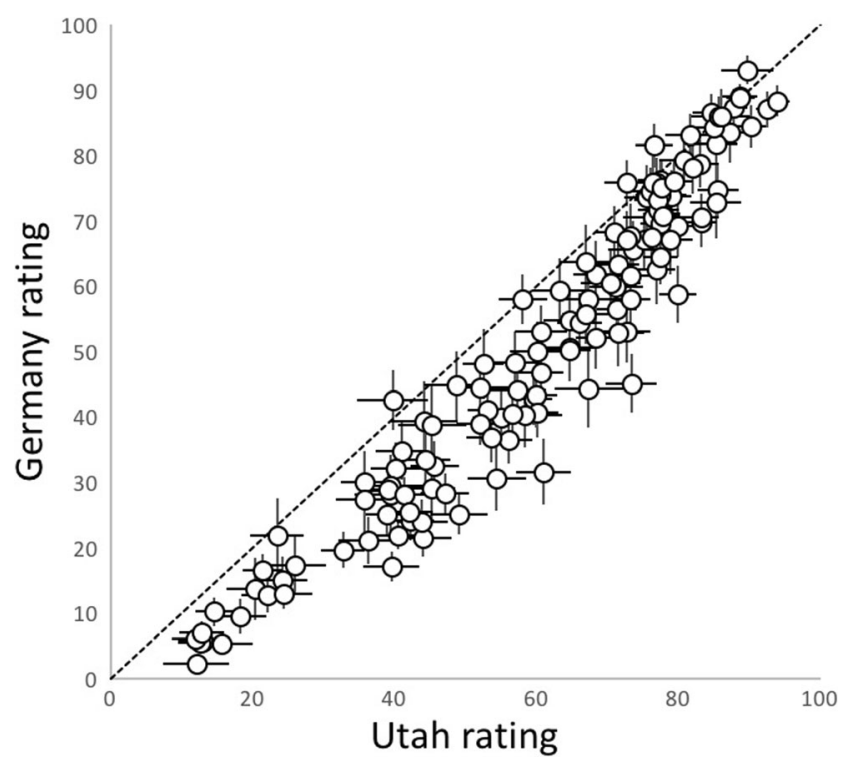

Fig. 2 Average rating for German vs. Utah participants by image. Vertical error bars represent \pm 1 German standard error, horizontal bars represent \pm 1 Utah standard error. The dashed line represents complete agreement
Within-image variability, shown by the standard error, was small relative to the variation in mean ratings across images. Importantly, intergroup agreement was indicated by a strong linear relationship between the two samples, $r(129)=0.92$, $p<0.001$, with a slope near unity (1.1). U.S. ratings were larger $(M=60.05, S D=10.16)$ than German ratings $(M=50.58, S D=10.07), t(49)=3.26, p=0.002$. The essentially constant difference could reflect the viewing angle or posture but also might reflect cultural variations. Thus, the study indicates that ratings of the largeness of depicted space in 2D images varying in natural content are reliable within and between groups. Although our participants rated largeness of depicted space, we interpret the perceptual dimension that underlies the regularities in their judgments as vastness.

There was clear variation in vastness across scene categories. Detailed analysis is deferred to Experiment 2, where the variables of openness and ruggedness are considered as well. For present purposes, we note that the categories judged most vast were scenes with a clear horizon line like an ocean and mountains and the least vast were forests and close views of vegetation.

These categories also emerged in the analysis of participants' recollections of vast experience. Their reports (in English except one translated from German) were first submitted to a lexical frequency count. The most frequent words (at least 10 reports), which tended to emerge in both countries, were look, feel, space, vast, large, ocean, small, see, mountain, and canyon. Items with greater than 5 occurrences $(\mathrm{N}=26$, excluding adverbs and high-frequency verbs) were submitted to latent semantic analysis (http://sa.colorado.edu/, data base General Reading up to first-year college), which provides a measure of inter-relatedness (cosine between word vectors, range 0 to \pm 1.0 ). The high-frequency items having a cosine value with vast of 0.3 or greater were: large, over, desert, mountain, time, ocean, small, horizon, and valley. Thus, both word count and similarity point to recollected vastness being associated with particular environmental entities and features, most notably those with horizon, height, or depth.

The structure emerging from both vastness ratings and recollected vast experiences echoes the categorical scaling of openness and ruggedness, where highly open stimuli were exemplified by coastlines and highly rugged stimuli by mountains, with forests low on both variables (Oliva \& Torralba, 2001). The finding that similar scene categories exemplify high levels on all three variables is consistent with the hypothesis that the sense of vast space may arise from scene-related processing. At the same time, this commonality raises the question of whether the descriptor "vast" adds unique qualities beyond "open" or "rugged." The question of how well vastness can be accounted for by these other scene-specific properties was directly addressed in Experiment 2. 


\section{Experiment 2: Relating vastness to scene parameters}

\section{Method}

\section{Participants}

Twenty-two (14 females, 8 males) University of Utah college students (mean age $=26$ years) participated for course credit. All gave written consent. Sample size and stopping rule were as in Experiment 1.

\section{Materials and Procedure}

The images from Experiment 1 were used in Experiment 2. The participant sat before a Dell U2412M monitor, at a distance such that each image subtended a visual angle of approximately $22^{\circ}$ wide by $15^{\circ}$ high.

The experimental task was to rate each image on two dimensions derived from computational scene analysis: openness and ruggedness (Oliva \& Torralba, 2001). Because verbal descriptions of open and rugged can lead to variable interpretations (Kravitz et al., 2011), we asked subjects to assign values to these properties by placing them within an openness/ruggedness space obtained from the original algorithm of Oliva and Torralba (2001). The dimensions were depicted by 64 representative grey-scale images of outdoor scenes, arranged in a 2D openness ( $\mathrm{x}$ axis) by ruggedness (y axis) grid, as shown in Fig. 3(Oliva, unpublished). Under the display conditions, the grid was approximately $19^{\circ}$ on each dimension. In addition to the visual depiction of the dimensions, participants were also given verbal definitions: openness was: "not having many obstructions or objects on the

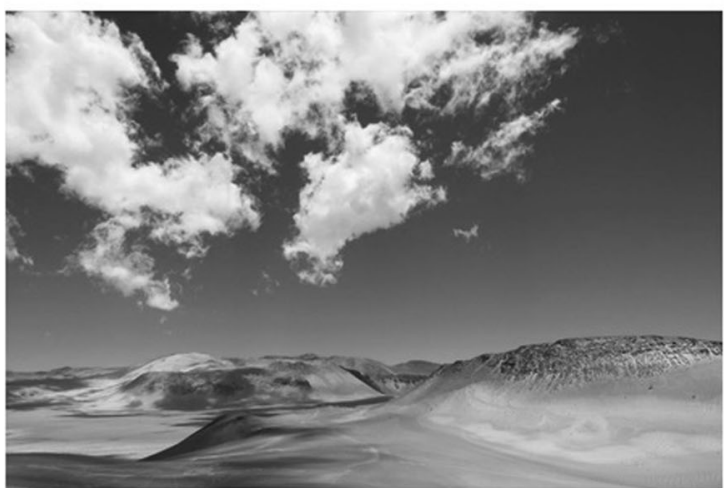

Click on the matrix of images on the right at the position that best represents where it falls along the open and rugged axes.

You can click on any position and are not restricted to clicking in the center of one of the individual images. ground surface,” and ruggedness was: “... protrusions or projections of ground surface into the height dimension, producing an uneven surface."

The 131 images rated in Experiment 1 were presented one at a time, in random order, each placed next to the opennessruggedness matrix. Participants were asked to point with the mouse to the location that best represented where the current environmental scene fit in terms of the two dimensions (Fig. 3). They clicked to record the location and advance to the next trial. The instructions specified that they were not trying to match to a particular image, but should respond on the basis of the underlying axes.

Prior to making the openness/ruggedness ratings, participants were pretrained on use of a similar grid, comprising an arrangement of four facial expressions representing variations in energy (x axis) and valence (negative to positive on y axis). They were asked to relate two new facial images to these dimensions, by using the mouse-click task to be performed in the experiment proper.

\section{Results}

An average openness and ruggedness value was calculated for each image on a $0-1$ scale; the means are shown for the two dimensions in Fig. 4 along with the standard error on each dimension. The variability for openness $(S D=0.21)$ was somewhat greater than for ruggedness $(S D=0.16)$. As noted previously, there is some dependence between these variables (high openness means low ruggedness) but unconstrained variation is at least possible in some regions of the scales. The linear correlation between the parameters for this image set was low $(r=0.01)$. There was, however, a trend toward a

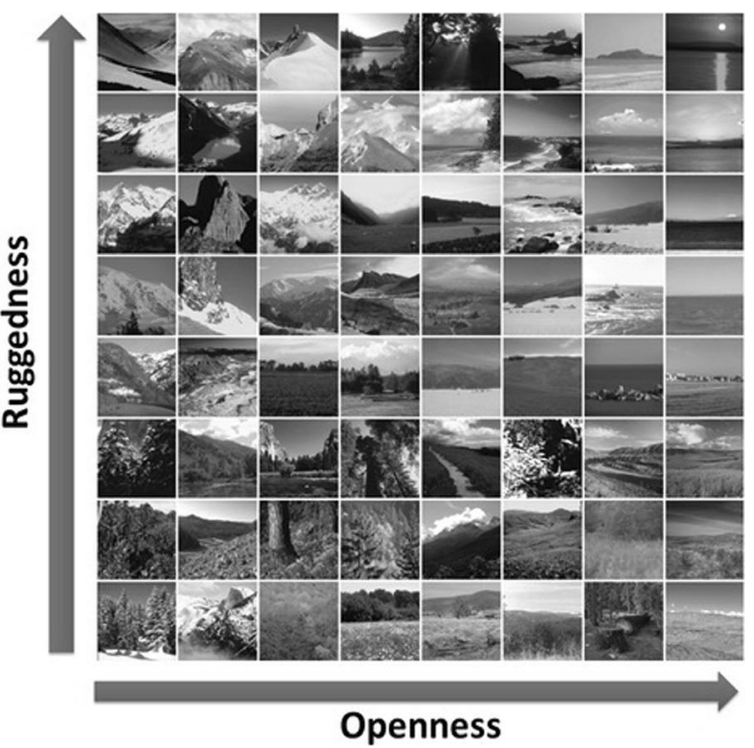

Fig. 3 Example trial for rating images as open and rugged. Image on the left retrieved from Pixabay. Sample and judged images (on left) were in color 


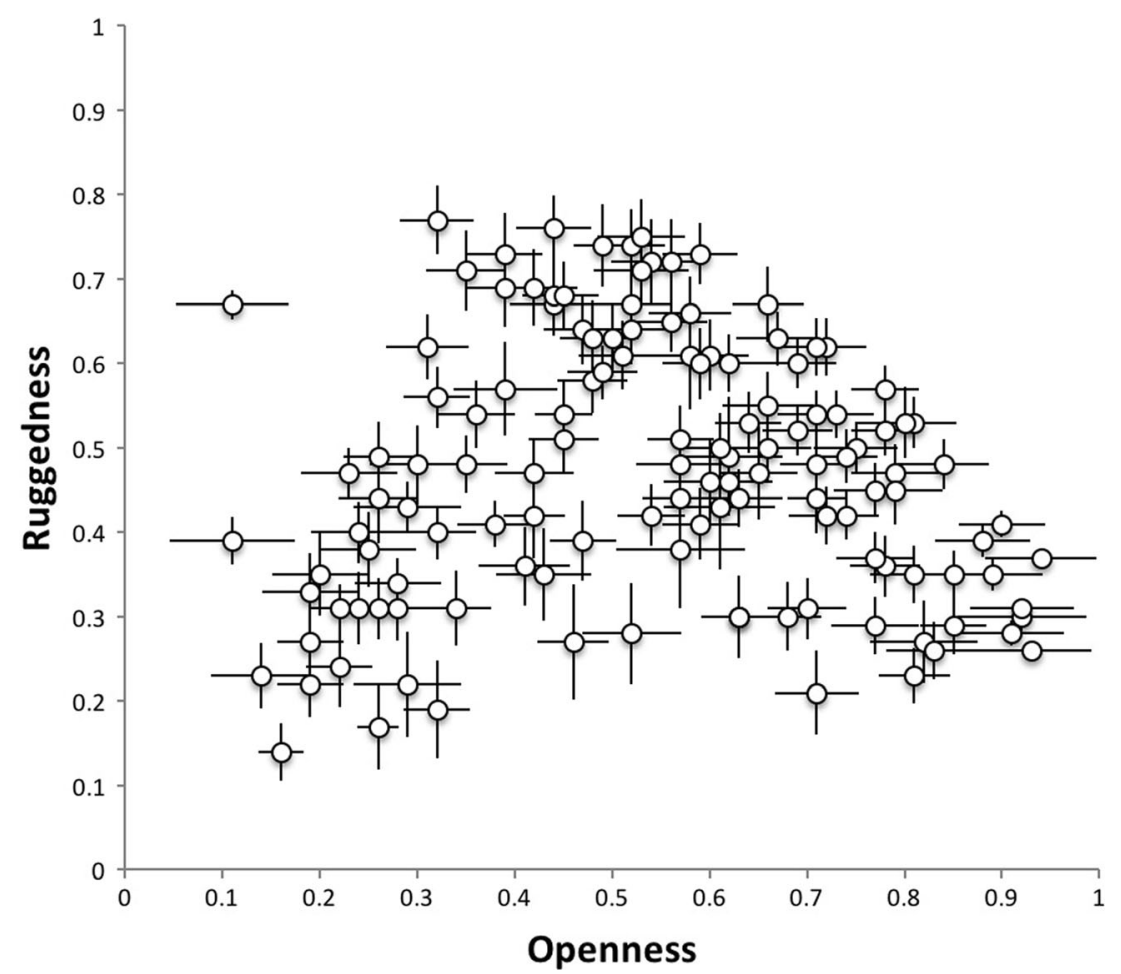

Fig. 4 Average rated ruggedness in relation to openness in Experiment 2, by image. Error bars represent 1 standard error for each dimension

curvilinear relationship (for a second-order polynomial fit, $R^{2}=0.28, p<0.001$ ), with ruggedness ratings tending to be highest for images that were moderate in openness. The importance of this relationship emerges in the subsequent analysis.

The principal analysis assessed how vastness, a perceptual response, was related to openness and ruggedness, variables derived from visual properties of scenes. Linear regression using the mean values for each image assessed openness and ruggedness as predictors of the largeness ratings in Experiment 1 (averaged across the two samples). When considered in separate bivariate regressions, both openness and ruggedness were significant predictors of largeness (for openness, $\beta=0.78, p<0.001, R^{2}=0.60$; for ruggedness, $\beta=0.46$, $p<0.001, R^{2}=0.21$ ). In an unforced stepwise multivariate regression with greater F-test criterion, openness was entered before ruggedness. As would be expected from the null correlation between the predictors, their coefficients were the same in the stepwise regression as when used in isolation ( $p \mathrm{~s}<0.001$ ). The $R^{2}$ increased from 0.60 in the model with openness as the sole predictor to $R^{2}=0.82$ for the multivariate model, indicating that ruggedness accounted for an additional $22 \%$ of the variance in largeness ratings after openness was controlled, amounting to $55 \%$ of the residual variance not explained by openness.

The contribution of ruggedness beyond openness can be seen in Fig. 5, which displays the residuals from the bivariate largeness/openness regression against ruggedness and portrays examples of specific images for which largeness is predicted versus over- or underpredicted by openness. What is apparent is that for those images where vastness was overpredicted by openness (i.e., where residuals are negative), ruggedness tends to be low. Conversely, where vastness is underpredicted by openness (i.e., where residuals are positive), ruggedness is high. For images rated at the mean of ruggedness, vastness is well predicted by openness (i.e., the values of residuals are very small).

Experiment 2 demonstrated that ratings of spatial largeness in a depicted scene, which we interpret as perceived vastness, are related to the scene-based categories of openness and ruggedness, which have categorical and spectral signatures. Although at least in our stimulus set, openness was a stronger predictor of perceived vastness, a striking aspect of the results is that the errors made when using openness alone to predict vastness were not random. It appears that images that are not particularly open but are judged to depict vast spaces and tend to be rugged, as in mountain scenes.

A further analysis explored the relation between specific image content and perceived vastness, using the categories identified in Experiment 1. Figure 6 shows the distribution of vastness, openness, and ruggedness by category across the 123 categorizable images (for all three variables, with vastness rescaled as in Fig. 6, standard deviations (SD) across images in a category ranged from 0.06 to 0.14 ). Vastness and its visual correlates show systematic variations across categories. The category with highest vastness, images with a salient horizon, also was rated most open. However, consistent with the results from the rating task of Experiment 2, openness 


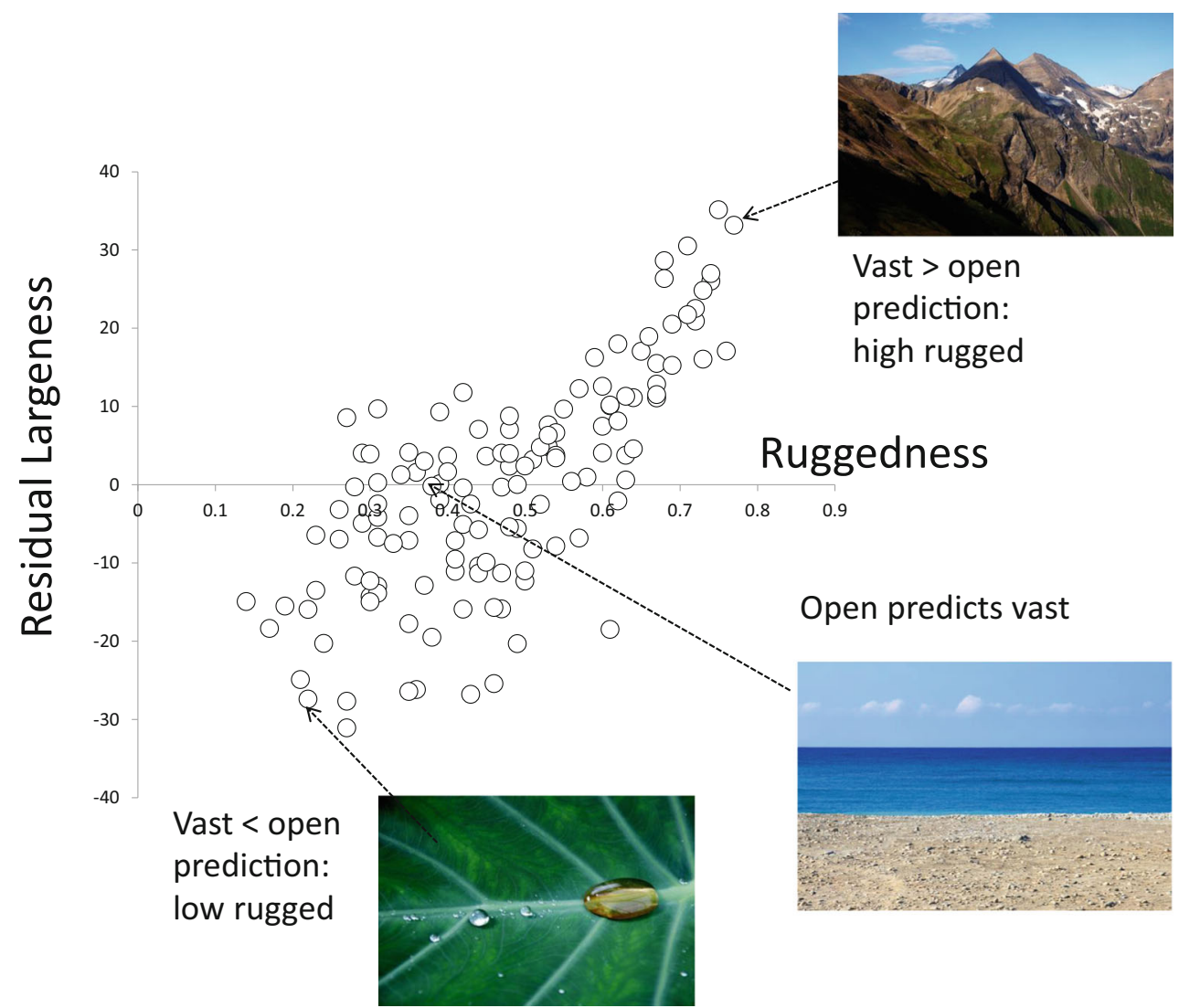

Fig. 5 Residuals after the prediction of largeness by openness, in relation to ruggedness, by image. Positive residuals indicate that openness underpredicted vastness, and negative residuals indicate that openness

alone did not predict vastness; mountain scenes, which were rated high in ruggedness but only moderate in openness, also were perceived as vast, and some open categories, such as

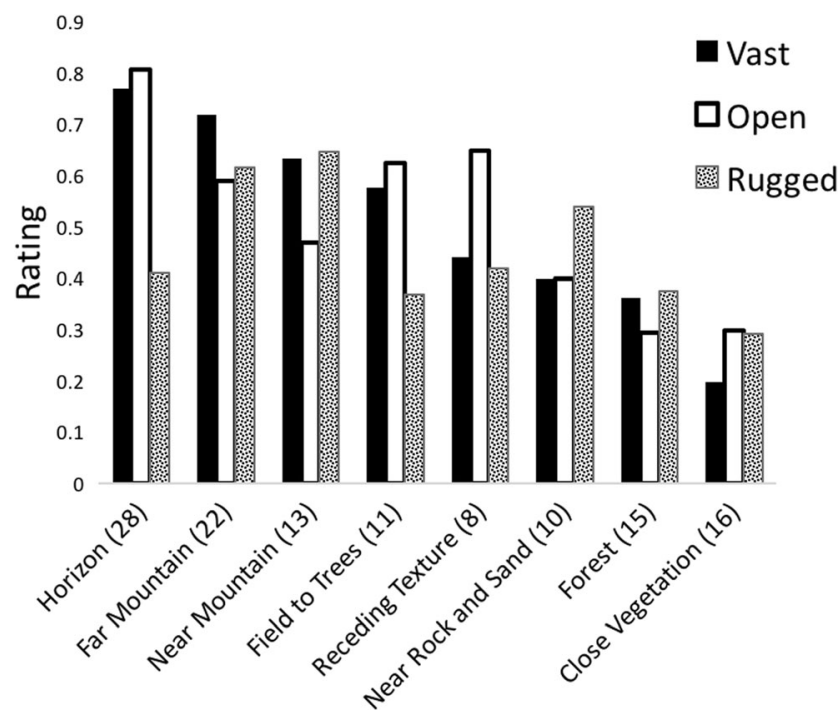

Fig. 6 Mean rating for vastness (divided by 100 to equate scale ranges), openness, and ruggedness, by category. The number of images for each category is shown with the label overpredicted vastness. The data points corresponding to the same scenes as in Fig. 1 are shown to illustrate the effects

receding textures, were not vast. The least vast categories were those with low openness and ruggedness.

\section{Experiment 3: Relating vastness to metric distance judgments}

One would expect vastness to have some relationship to distance in depth, given that vast spaces were defined in terms of spatial extent. Our approach postulates, however, that the sense of vast space cannot be based directly on knowledge of distance in metric terms, because metric depth cues lack reliability at the scale of spaces considered vast. In a final experiment, we further tested the contribution of distance to vastness by asking people to make judgments of metric depth of the previous image set. Specifically, they judged the distance to the center of the space in a metric of their own choice. This response measure was adapted from Torralba and associates (2002), who obtained metric values (in meters) of "mean depth" of 400 pictured spaces from observers' judgments. When extrapolated to a larger set of 4,000 ranked images, mean depth was found to be predicted from scaledependent measures of image structure, which was taken as support for its use as a measure of metric spatial extent. 


\section{Method}

The subjects were 29 naïve participants from the same pool as Utah participants in Experiment 1. One subject was ultimately eliminated, due to deviant responses (individual mean $>5$ SD from the overall mean distance). An initial induction, intended to make participants think of themselves as spatial observers, asked them to "recall an experience of looking out at a space of near or far objects and surfaces, making you feel like an object at the edge of the space." They then proceeded to judge the sequence of images used previously, with the exclusion of one image that depicted only sky. The instructions told participants to think of themselves as taking the picture and, from what they would see, to report "the distance from the photographer to the center of the depicted objects and surfaces in the image (not the sky)." They indicated the distance value and the metric they were using (e.g., yards) for each judgment. All responses were converted to meters.

\section{Results and Discussion}

Two images taken from a viewpoint high above the ground plane produced distance ratings of $>90,000$ meters, more than 3 SD from the mean across images (the corresponding vastness ratings were 77 and 90). These outliers were eliminated from further quantitative assessment of distance as a predictor of Utah largeness ratings. The average distance ratings for the remaining 128 images ranged from $0.06 \mathrm{~m}$ to $69,716 \mathrm{~m}$ with a mean of $12,130 \mathrm{~m}$. Largeness was positively correlated with distance across images $\left(R^{2}=0.51, p<0.001\right)$. However, as shown in Fig. 7, a power function of largeness versus distance accounted for considerably more variance $\left(R^{2}=0.83\right)$. Notably, the exponent was 0.13 , reflecting a striking compression of largeness across the distance scale. Metric distance and largeness also differed with respect to predictability from ruggedness and openness: a stepwise regression of distance with openness as the sole predictor found $R^{2}=0.25$, increasing only to $R^{2}=0.37$ for the model with ruggedness also included. This is well below the $R^{2}=0.82$ obtained for the corresponding regression of largeness. A further regression addressed whether distance predicted largeness beyond openness and ruggedness. An unforced stepwise regression entered openness first, then ruggedness (i.e., the same model as the previous), and finally distance, which increased the $R^{2}$ only by 0.05 .

Finally, it should be noted that while most participants were able to rate most images sufficiently that the mean distance was significantly correlated with mean largeness, the intersubject variability was high, as indicated by an average coefficient of variation (SD scaled by mean) of 2.4. For reference, the mountain image in Fig. 1 had an average rating of approximately 18 miles but was judged from within a mile to 150 miles away. Moreover, because the SD was highly correlated with the mean $(r=0.95)$, distance judgments were most unreliable for the very spaces perceived to be vast.

The present findings offer little support for the idea that the vastness percept is mediated by an estimate of the distance of visible features and surfaces. If so, substantial inflation at large distance values would be required for the observed mapping of distance into the largeness scale. The further finding that

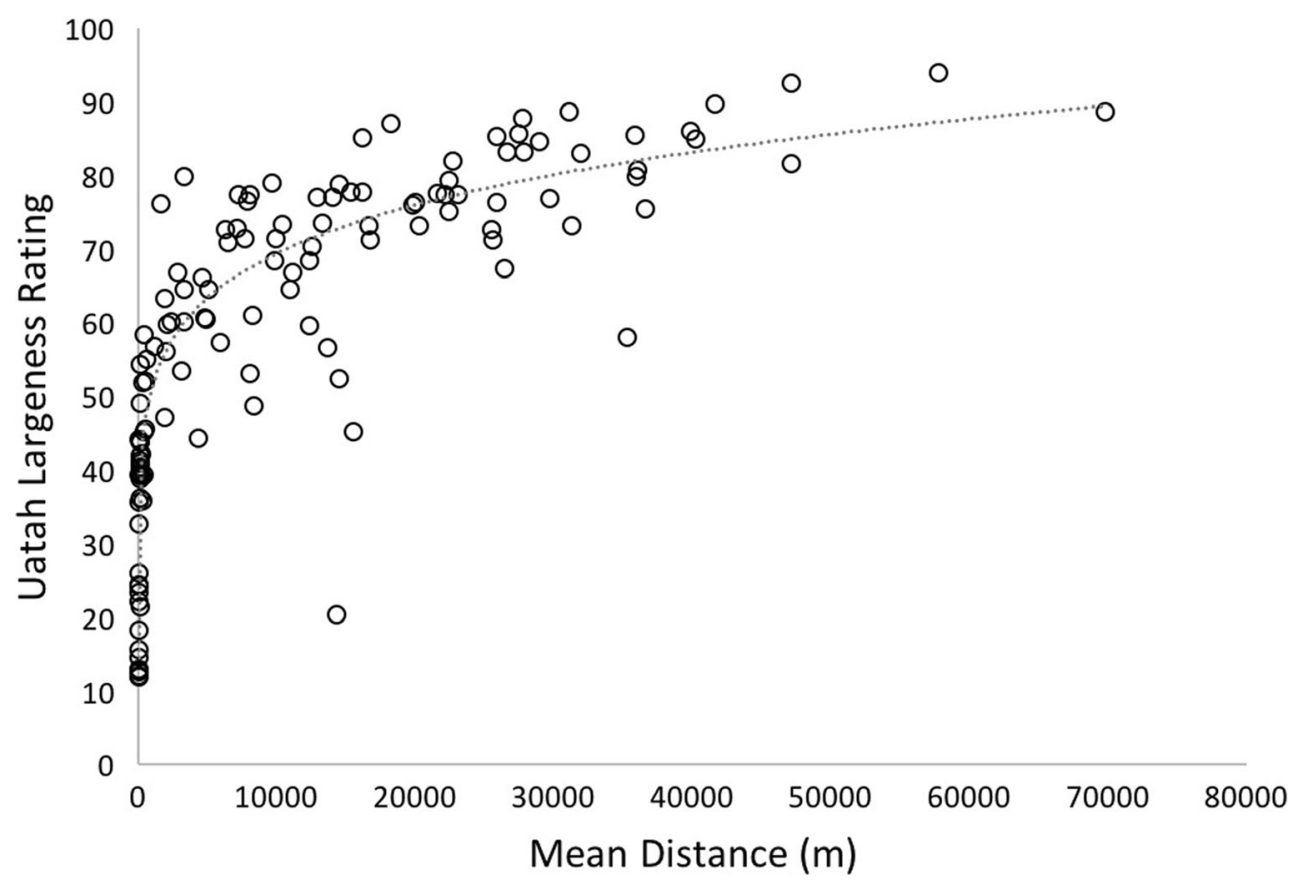

Fig. 7 Average rated largeness by Utah participants in Experiment 1, in relation to average distance judgments in Experiment 3, by image. A power function fit is shown 
distance, unlike largeness, is not well predicted by openness and ruggedness, together with the obvious difficulty of the metric distance task - not surprising given the paucity of cues - suggest that the perceived extent of surrounding space is not based on processes that yield metric depth perception.

\section{Discussion}

The present results indicate that the impression of vast space arising from pictorially presented scenes is a reliable phenomenon. The consistency of ratings of spatial extent point to commonalities in the perception of vastness across individuals within and between cultures and under varying display conditions. Given that these judgments were based on 2D images of natural scenes, ranging over many orders of magnitude in geometric scale, it appears that people do not have to be physically present or immersively projected into a space to judge that it is vast.

Experiment 2 further pointed to geometric and spectral features underlying a sense that an environment is vast. Rated largeness of scenes was robustly related to their openness and ruggedness. Neither of these properties guaranteed a sense of vastness, but the data indicated that natural environments lacking in both will not be evocative of spatial extent. Of the two parameters, openness was a better predictor of perceived spatial extent. Its greater importance is consistent with evidence that cortical areas specialized for scene perception also are sensitive to openness (Kravitz et al., 2011; Park et al., 2011).

These results raise the question of whether vastness identifies some unique aspect of scene perception beyond openness and ruggedness. If the explained variance between German and U.S. ratings $\left(r^{2}=0.85\right)$ identifies a target for reliable prediction, the vastness/openness regression $\left(r^{2}=0.60\right)$ accounts for $70 \%$ of this goal, and openness and ruggedness together account for $96 \%$ (multiple $\mathrm{R}^{2}=0.82$ ). However, describing vastness as a weighted combination of openness and ruggedness overlooks the systematic nature of the prediction, which suggests multiple routes to the vast experience. Spaces described as large can be extremely open or they can be moderately open but rugged. The linear prediction also fails to account for an essential feature of vastness - the sense that space extends from the viewer without limit.

How this sense of distance is obtained in vast spaces remains an intriguing question. These results indicate that people discriminate degrees of vastness in scenes where much or all of the terrain is viewed at distances where traditional metric depth cues cease to be informative, according to theory and as supported by the findings of Experiment 3. Likewise, openness and ruggedness are parameters that do not in and of themselves provide direct estimates of environmental distance and scale. We speculate that the impression of distance may arise from a combination of relative depth information in the presented scenes (that cannot on its own specify scale) and further, as yet unspecified, scene properties. For example, an abundance of interposition cues, while failing to support metric distance perception, might allow a scene to perceptually extend in depth essentially without limit. Categorical assignment might also contribute at a semantic level.

Various features of scenes could enhance this experience. One approach, a common compositional convention in nature photography, is to use foreground structures that are within the effective range of standard depth cues and can provide a contrast to the unknowably farther distances associated with a large-scale background. Another influential compositional effect is height in the picture plane (corresponding to angle of gaze in the real world), as in looking up at a mountain from below or looking down on a canyon from above. The contribution of the horizon line to rated vastness could indicate that features arising from perspective structure play an important role in the perception of vastness. Perspective is unlikely to be the whole story, because small-scale (i.e., non-vast) environments can also generate strong perspective cues. A limitation of our study is that by using photographs from arts websites, we could not control perspective or enhancements or identify their contributions. Indeed, given that stimulus selection was intended to induce variations in perceived largeness, it seems inevitable that the present vast scenes exemplify conventions meant to enhance the sense of space.

A further possibility is that the perceptual mechanisms involved in vastness are statistical in nature, rather than being salient features or compositional effects. This would be consistent with findings of an association between image statistics and human judgments of scene depth (Torralba \& Oliva, 2002, although this work did not distinguish between metric distances vs. a more general concept of spatial extent). A statistical approach is supported by the contributions of openness and ruggedness to vastness, because these features have both been shown to be predictable based on 2D spectral image statistics (Oliva \& Torralba 2001). Ultimately, the perception of vastness may be modeled by multiple-pathway architecture, such as the combination of image statistics and features, as has been theorized more generally in scene perception (Wolfe et al., 2011).

To our knowledge, the current experiments are the first to show consistency in the types of environmental scenes that people rate as vast and to demonstrate that vastness is related to image-based aspects and conceptual categories of scenes. The importance of these results, we would argue, goes beyond their perceptual implications and the theoretical questions they raise, because vastness appears to be something more than a purely perceptual outcome. An expanded sense of space has been implicated as a precursor to the emotion of awe, which in turn has been linked to well-being, social engagement, and helping behaviors (Rudd, Vohs, \& Aaker, 2012; Van Cappellen \& Saroglou, 2012). Alterations of perceived space 
outside of the body also are associated with meditative relaxation practice (Berkovich-Ohana et al., 2013). These tantalizing connections suggest that a better understanding of the mental state induced by vast environments could have farranging implications.

Acknowledgements The authors thank Betty Mohler, who hosted three of us for some of the research conducted and who contributed to the conceptualization of vastness, and Aude Oliva, who provided the matrix used in the instructions of Experiment 2. JKS and RLK also acknowledge support from the Alexander von Humboldt Foundation, and RK from NSF grant IIS-1518630.

\section{References}

Berkovich-Ohana, A., Dor-Ziderman, Y., Glicksohn, J., \& Goldstein, A. (2013). Alterations in the sense of time, space, and body in the mindfulness-trained brain: A neurophenomenologically guided MEG study. Frontiers in Psychology, 4, 912.

Cutting, J. E., \& Vishton, P. M. (1995). Perceiving layout and knowing distances: The interaction, relative potency, and contextual use of different information about depth. In W. Epstein \& S. Rogers (Eds.), Perception of Space and Motion (pp. 69-117). San Diego: Academic Press.

Finn, R. H. (1970). A note on estimating the reliability of categorical data. Educational and Psychological Measurement, 30, 71-76.

Greene, M. R., \& Oliva, A. (2009). The briefest of glances: the time course of natural scene understanding. Psychological Science, 20(4), 464-472.

Henderson, J. M., \& Hollingworth, A. (1999). High-level scene perception. Annual Review of Psychology, 50, 243-271.
Joubert, O. R., Rousselet, G. A., Fize, D., \& Fabre-Thorpe, M. (2007). Processing scene context: Fast categorization and object interference. Vision Research, 47(26), 3286-3297.

Kravitz, D. J., Peng, C. S., \& Baker, C. I. (2011). Real-world scene representations in high-level visual cortex: It's the spaces more than the places. Journal of Neuroscience, 31, 7322-7333.

Loomis, J. M., \& Knapp, J. M. (2003). Visual perception of egocentric distance in real and virtual environments. In L. J. Hettinger \& M. W. Haas (Eds.), Virtual and Adaptive Environments (pp. 21-46). Mahwah: Erlbaum.

Oliva, A., \& Torralba, A. (2001). Modeling the shape of the scene: A holistic representation of the spatial envelope. International Journal of Computer Vision, 42, 145-175.

Park, S., Brady, T. F., Greene, M. R., \& Oliva, A. (2011). Disentangling scene content from its spatial boundary: Complementary roles for the PPA and LOC in representing real-world scenes. Journal of Neuroscience, 31, 1333-1340.

Rudd, M., Vohs, K. D., \& Aaker, J. (2012). Awe expands people's perception of time, alters decision making, and enhances well-being. Psychological Science, 23, 1130-1136.

Siegl, B., Livesey, E., Browne, L. (2005). Examples of stimulus photographs (originally in color). The leaf and shore images were retrieved from Pixabay, http://creativecommons.org/licenses/by/3.0/. The mountain image was retrieved from DeviantArt http:// austriaangloalliance.deviantart.com/art/Austrian-Peaks-328637716.

Torralba, A., \& Oliva, A. (2002). Depth estimation from image structure. IEEE Transactions on Pattern Analysis and Machine Intelligence, 24, 1-13.

Van Cappellen, P., \& Saroglou, V. (2012). Awe activates religious and spiritual feelings and behavioral intentions. Psychology of Religion and Spirituality, 4, 223-236.

Wolfe, J. M., Võ, M. L.-H., Evans, K. K., \& Greene, M. R. (2011). Visual search in scenes involves selective and non-selective pathways. Trends in Cognitive Sciences, 15, 77-84. 